\title{
Rede Neural Artificial Convolucional Aplicada ao Reconhecimento de Configuração de Mão nos Símbolos de 0 a 9 da Língua Brasileira de Sinais (LIBRAS)
}

\author{
Adriel Vieira Santos ${ }^{1}$, Iago Franco Bacurau ${ }^{1}$, Jayne de Morais Silva ${ }^{1}$, \\ Talles Brito Viana ${ }^{1}$, Robson Gonçalves Fechine Feitosa ${ }^{1}$ \\ ${ }^{1}$ Instituto Federal de Educação, Ciência e Tecnologia do Ceará \\ Rodovia CE 292, KM 15, Gisélia Pinheiro - CEP 63115-500 - Crato - Ceará - Brasil \\ adriel.klt@gmail.com, \{tallesbrito,robsonfeitosa\}@ifce.edu.br
}

\begin{abstract}
Brazilian Law number 10.436, of April 24th, 2002, recognizes the Brazilian Sign Language (LIBRAS) as a legal way for communication and expression. However, the non-listening community, which makes use of this language, has difficulties to communicate with the listening community. In order to decrease such a difficulty, in this work is described an application of a convolutional neural network for the LIBRAS static symbols recognition. To validate the model, a database with about 2640 images was used, including symbols from 0 to 9 for training; and, 1360 symbols for testing. In addition to an extra set of 1000 symbols for validating more test cases. As a result, a recognition rate ranging from $82.5 \%$ to $98.57 \%$ for different symbols was obtained.
\end{abstract}

\begin{abstract}
Resumo. A Lei brasileira número 10.436, de 24 de abril de 2002, reconhece a Língua Brasileira de Sinais (LIBRAS) como meio legal de comunicação e expressão. Entretanto, a comunidade não ouvinte, que faz uso dessa língua, possui muita dificuldade de comunicação com a comunidade ouvinte. Visando reduzir tal dificuldade, o presente trabalho descreve uma aplicação das redes neurais convolucionais para o reconhecimento dos símbolos estáticos da LIBRAS. Para validar o modelo, foi utilizada uma base de dados com cerca de 2640 imagens, com símbolos de 0 a 9 para treinamento; e, 1360 símbolos para teste. Além de um conjunto extra de 1000 símbolos para validação de mais casos de testes. Como resultados, obteve-se uma taxa de acerto que varia entre $82.5 \%$ e $98.57 \%$ para os diferentes símbolos.
\end{abstract}

\section{Introdução}

De acordo com [de Freitas et al. 2017], o censo do IBGE (realizado em 2010) mostra que 45,6 milhões de brasileiros possuem algum tipo de deficiência auditiva e, devido a lei $\mathrm{n}^{\circ} 10.436$, a Língua Brasileira de Sinais (LIBRAS) foi reconhecida como uma língua oficial no Brasil. Dessa forma, o tema de acessibilidade e suas tecnologias é abordado em vários trabalhos, como: [Brandt 2015] e [Koroishi and Silva 2015]. Diante disso, o presente trabalho possui como objetivo demonstrar a viabilidade do uso das redes neurais convolucionais para o reconhecimento de alguns dos símbolos estáticos da LIBRAS (números de 0 a 9) capturados por vídeo.

As RNAs (Redes Neurais Artificiais) são modelos matemáticos inspirados nos neurônios biológicos. Elas são aplicadas na resolução de problemas que envolvem: 
desempenho, poder de generalização, robustez e tolerância a falhas. Devido suas características inspiradas nos neurônios biológicos, sua aplicação na identificação de padrões em imagens foi um caminho natural, em que, por exemplo (de acordo com [Haykin 2007]), uma pessoa consegue identificar um rosto familiar em uma cena qualquer em cerca de 100 à 200 milissegundos.

Outro fator interessante para uso das redes neurais artificiais, como solução ao reconhecimento de padrões em imagens, é a aplicação em problemas em que não é possível descrever em detalhes um modelo matemático formal (algorítmico) como solução. Por conta dessa característica, [Barreto 2002] visualiza as RNAs como um paradigma para resolução de problemas.

Para fins de organização, o presente trabalho está dividido nas seguintes seções: a Seção 2 apresenta a fundamentação teórica que embasa o trabalho; na Seção 3 descrevese a metodologia e materiais utilizados; e, por fim, a Seção 4 ilustra os resultados e conclusões encontradas.

\section{Fundamentação Teórica}

\subsection{Redes Neurais Artificiais}

Segundo [Haykin 2007], uma RNA é um processador maciçamente paralelamente distribuído consistindo de unidades de processamento simples, que têm a propensão natural para armazenar conhecimento experimental e torná-lo disponível para o uso.

As redes neurais artificiais podem ser visualizadas em duas arquiteturas diferentes: redes alimentadas para frente e redes recorrentes. O primeiro tipo de rede pode ser visualizada como um grafo ${ }^{1}$ acíclico dirigido e o segundo tipo como um grafo cíclico dirigido em que a saída de um neurônio pode ser utilizada para alimentar o próprio neurônio novamente ou toda a rede. A rede neural pode conter várias camadas. Quanto maior a quantidade de camadas, maior a capacidade de resolução de problemas da rede.

A rede neural Multilayer Perceptron (MLP) é um tipo de rede neural que deriva do Perceptron desenvolvido por Rosenblatt em 1958. De acordo com [Haykin 2007], este é o modelo mais simples de rede neural para tratar problemas linearmente separáveis como os operadores $O U$ e $E$ da lógica. Além disso, segundo o mesmo autor, Rosenblatt demonstra com sua pesquisa um teorema que garante a separação de problemas lineares por redes do tipo Perceptron simples. O treinamento das redes do tipo MLP utiliza o algoritmo Backpropagation. Segundo [Haykin 2007], este algoritmo é categorizado como aprendizagem por correção de erro.

Segundo [Vargas et al. 2016], uma Rede Neural Convolucional (RNC) é uma variação das redes MLP, inspirada no processo biológico de processamento de dados visuais. A estrutura desse tipo de rede pode ser dividida em duas partes: camadas para extração de características e camadas de classificação.

De acordo com [Vargas et al. 2016], uma convolução é uma operação entre duas funções que produz uma terceira função. O objetivo da aplicação da convolução na rede é uma forma de integrar sobre o modelo a capacidade de extrair informações dos campos

\footnotetext{
${ }^{1}$ Um grafo é uma entidade formada por um conjunto de arestas e um conjunto de vértices, sendo as arestas uma relação binária sobre o conjunto de vértices.
} 
perceptíveis contidos nas imagens, assim como ocorre na visão humana. Acredita-se que quando uma pessoa visualiza uma imagem, ela não a interpreta como um todo, mas sim em partes que a caracterizam.

Baseado em [Mansano 2017] e [Vargas et al. 2016], para entender o funcionamento da rede é necessário entender a etapa de convolução. Dado uma imagem binarizada, representada em uma matriz bidimensional, pode-se aplicar sob esta uma outra matriz que representa um padrão que se deseja extrair, também chamada de kernel. A aplicação da convolução entre essas duas funções produz uma nova matriz que fornece evidências do padrão contido na imagem. Após a convolução, aplica-se uma técnica de pooling (máximo, mínimo, média ou mediana) no conjunto de elementos contidos dentro da área de um kernel. A utilização dessa última técnica permite que as alterações ocorridas na imagem como rotação, escala e translação se tornem invariantes.

$\mathrm{O}$ algoritmo de aprendizagem Backpropagation é utilizado para treinar a rede. Por conta da grande quantidade de camadas que podem existir, é necessário utilizar nas camadas de extração de características a função de ativação ReLU (unidade linear retificada), que evita o problema do desaparecimento do gradiente. Diferente das funções sigmoide e tangente hiperbólica, como mostrado em [Academy 2018].

\section{Metodologia}

Para a construção do modelo proposto neste trabalho, foi necessária a utilização do WEKA. Segundo [Frank and Mark 2016], o WEKA é uma coleção de algoritmos de aprendizado de máquina para tarefas de mineração de dados. Esta contém ferramentas para preparação de dados, classificação, regressão, agrupamento, mineração de regras de associação e visualização. A escolha desta ferramenta foi motivada por conter a implementação do modelo apresentado, ser open-source e dispor de métodos de avaliação dos classificadores e sua visualização.

Tendo como objetivo testar o modelo, foi necessário produzir uma base de dados contendo 4000 imagens com tamanhos $28 \times 28$ possuindo os símbolos de 0 a 9 da língua brasileira de sinais (LIBRAS). As imagens foram obtidas de uma câmera VGA integrada em um notebook Positivo, em um ambiente com fundo branco. A imagem foi binarizada usando a classe BufferedImage (biblioteca disponível na API da linguagem de programação Java) ${ }^{2}$.

A rede neural convolucional treinada segue a arquitetura LeNet ${ }^{3}$. Tal arquitetura obteve sucesso no reconhecimento de dígitos manuscritos, conforme apresentado em [LeCun et al. 1995]. Outro motivo que embasou a escolha da arquitetura LeNet foi pelo bom desempenho, mesmo em máquinas de baixa capacidade de processamento, como a que foi utilizada para o treinamento do presente trabalho. Os parâmetros usados na rede foram:

- Taxa de aprendizado: 0.001; Tamanho do lote: 120; Função de ativação: ReLU; Número de épocas: 10; Camadas de convolução: 02, sendo: Camada 01 com 20 neurônios e Camada 02 com 50 neurônios; Camada oculta (MLP interna) com 500 neurônios; Camada de saída com 10 neurônios; e, Número de pooling: 02.

\footnotetext{
${ }^{2}$ Disponível em: https://docs.oracle.com/javase/7/docs/api/java/awt/image/ BufferedImage.html.

${ }^{3}$ Disponível em: http: / /yann. lecun. com/exdb/lenet/.
} 


\section{Resultados e Conclusões}

A rede neural foi treinada com 2640 imagens, referentes a cerca de $66 \%$ da base de dados, sendo que as 1360 imagens restantes foram usadas para testes, cerca de $34 \%$. O classificador consegui obter uma taxa de acerto de $98.57 \%$ (1343 imagens). Isso demonstra que os resultados foram muito positivos para o conjunto usado.

Devido à baixa capacidade da máquina disponível, não foi usada a técnica de validação cruzada, descrita em [Haykin 2007], durante a avaliação do modelo. Assim, um importante trabalho futuro seria realizar uma análise mais apurada dos resultados, com o intuito de verificar se há indícios de super adaptação. Para contornar este problema, uma nova base de dados foi criada por uma única pessoa. A base foi formada por 1000 imagens e a LeNet ainda classificou corretamente cerca de $82.5 \%$ das instâncias de forma correta.

Embora os resultados alcançados sejam relevantes, é importante notar que a produção de uma base de dados mais diversificada com uma alta quantidade de imagens pode, possivelmente, melhorar os resultados. Como a máquina disponível para o treinamento não possuía alta capacidade de processamento, não foi possível avaliar diferentes parâmetros e algoritmos com intuito de justificar a estrutura produzida.

\section{Referências}

Academy, D. S. (2018). Deep Learning Book, volume 1. Disponível em: deeplearningbook.com.br. Acesso em: 30 nov. 2018.

Barreto, J. M. (2002). Introdução às redes neurais artificiais. Florianópolis: UFSC.

Brandt, A. H. (2015). Lirann: sistema de reconhecimento de libras baseado em redes neurais artificiais com kinect. Disponível em: http://hdl.handle.net/11624/1031, acesso. Acesso em: 02 abr. 2019.

de Freitas, G. R., Maranhão, T. L. G., and Félix, W. M. (2017). A deficiência auditiva e a possibilidade de inclusão no mercado de trabalho. ID ON LINE REVISTA MULTIDISCIPLINAR E DE PSICOLOGIA, 11(34):184-218.

Frank, E. and Mark, A. (2016). Hall, and ian h. witten (2016). the weka workbench. online appendix for"data mining: Practical machine learning tools and techniques. Morgan Kaufmann, Fourth Edition., 78(71):74-5.

Haykin, S. (2007). Redes neurais: princípios e prática. Bookman Editora.

Koroishi, G. O. and Silva, B. V. L. (2015). Reconhecimento de sinais da libras por visão computacional. Mecatrone, 1(1).

LeCun, Y., Jackel, L., Bottou, L., Brunot, A., Cortes, C., Denker, J., Drucker, H., Guyon, I., Muller, U., Sackinger, E., et al. (1995). Comparison of learning algorithms for handwritten digit recognition. In International conference on artificial neural networks, volume 60, pages 53-60. Perth, Australia.

Mansano, A. F. (2017). O que é uma rede neural convolucional? Disponível em: https://www.linkedin.com/pulse/o-que-\%C3\%A9-um-rede-neural-convolucionalalex-fernandes-mansano, acesso. Acesso em: 30 nov. 2018.

Vargas, A. C. G., Paes, A., and Vasconcelos, C. N. (2016). Um estudo sobre redes neurais convolucionais e sua aplicação em detecção de pedestres. In Proceedings of the XXIX Conference on Graphics, Patterns and Images, pages 1-4. 